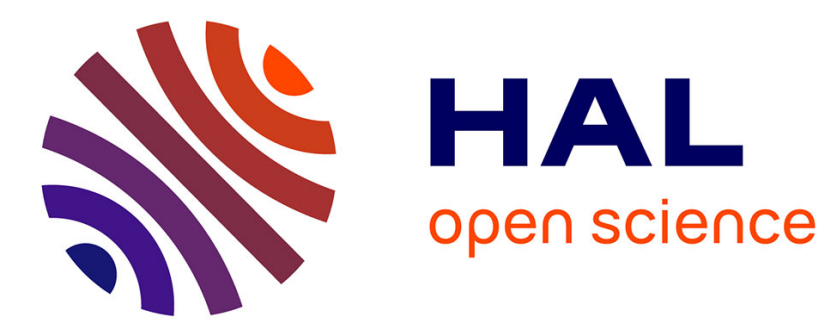

\title{
SOUDABILITE DE MATERIAUX SEMBLABLES ET DISSEMBLABLES
}

\author{
B. Petesch, L. Druette, R. Schmid, M. Robin
}

\section{To cite this version:}

B. Petesch, L. Druette, R. Schmid, M. Robin. SOUDABILITE DE MATERIAUX SEMBLABLES ET DISSEMBLABLES. Journal de Physique Colloques, 1987, 48 (C7), pp.C7-183-C7-184. 10.1051/jphyscol:1987734 . jpa-00227038

\section{HAL Id: jpa-00227038 https://hal.science/jpa-00227038}

Submitted on 1 Jan 1987

HAL is a multi-disciplinary open access archive for the deposit and dissemination of scientific research documents, whether they are published or not. The documents may come from teaching and research institutions in France or abroad, or from public or private research centers.
L'archive ouverte pluridisciplinaire HAL, est destinée au dépôt et à la diffusion de documents scientifiques de niveau recherche, publiés ou non, émanant des établissements d'enseignement et de recherche français ou étrangers, des laboratoires publics ou privés. 


\title{
SOUDABILITE DE MATERIAUX SEMBLABLES ET DISSEMBLABLES
}

\author{
B. PETESCH, L. DRUETTE, R. SCHMID et M. ROBIN \\ CALFETMAT/TUT, 17, Rue de France, F-69100 Villeurbanne, France
}

\begin{abstract}
Résumé : - Le soudage laser de feuilles de nickel, de tubes en Inconel et de feuflles de nickel sur de l'acier inoxydable a été étudié. Pour le dernier cas, malgré l'inhomogénéitê de la zone fondue, des résultats satisfaisants ont été obtenus en controlant la focalisation, la puissance et la vitesse de soudage.
\end{abstract}

Abstract : - LASER welding of nickel sheets, inconel pipes and nickel sheet to stainless steel plate has been studied. In spite of chemical inhomogeneity in the melting zone for the last case, satisfactory results could be obtained in manufacturing if focalisation, power and welding speed are carefully controlled.

Le travail prêsenté a poux but d'étudiex la faisabilité de divers types d'assemblages en vue d'applications industrielles.

\section{CONDITIONS OPERATOIRES}

Les sources sont des LASERS $\mathrm{CO} 2$ continus CILAS à flux axia1 rapide dont la puissance de sortie peut atteindre :

- $1000 \mathrm{~W}$ (CI 1000)

-3200 W (CI 4000)

pour des diamètres respectifs de faisceau de $l^{\prime}$ ordre de $12 \mathrm{~mm}$ et $20 \mathrm{~mm}$. La lentille, de focale $127 \mathrm{~mm}$, concentre ces faisceaux en une tache de 1 'ordre de $0,25 \mathrm{~mm}$. La protection gazeuse endroit est assurêe par injection latérale d'hélium dans le plan de joint. Les paramètres étudiés sont ceux qui influent le plus sur la morphologie de la zone fondue:

- distance entre tache focale et surface de la pièce (focalisation)

- puissance incidente.

- vitesse relative faisceau pièce.

\section{ASSEMBLAGES DE MATERIAUX SEMBLABLES}

Une première catégorie d'assemblages met en jeu des tôles d'épaisseur 0,5 et $1 \mathrm{~mm}$ en nickel pur en vue de la fabrication d'électrodes pour électrolyse de l'eau. Les tôles accostées et positionnēes par des outillages spécifiques, sont soudées soit bout à bout, solt par transparence, en " $\mathrm{T}$ " ou superposées. La plupart des essais sont réalisës avec une puissance de $800 \mathrm{~W}$. Les meilleurs résultats correspondent à des vitesses de 1 'ordre de $2 \mathrm{~m} / \mathrm{mn}$.

En soudage bord à bord, une protection envers est nécessaire ; dans le soudage en "I", les coupes transversales comportent un ventre (figure 1) situé dans la tôle verticale près de l'interface. Dans les trois cas envisagés, les assémblages réalisés conviennent pour les applications visẻes. Enfin, nous avons effectué quelques tests de soudure bout à bout de petits tubes en inconel (diamètre extérieur 19,1 mm, épaisseur $1,1 \mathrm{~mm}$ ) entrant dans $1 \mathrm{a}$ fabrication d'échangeurs. Vitesse et puissance sont comparables aux valeurs précêdentes. Les soudures sont toutes d'un bel aspect, malgré des valeurs de focalisation allant de $-1,5 \mathrm{à}+1 \mathrm{~mm}$. Les problèmes d'amorçage et d'évanouissement n'ont pas été étudiés. 


\section{ASSEMBLAGES DE MATERIAUX DISSEMBLABLES}

Une tôłe de nickèl d'épaisseur $0,5 \mathrm{~mm}$ a êté soudẻe par transparence sur une latte d'acier inoxydable $\mathrm{Z} 2 \mathrm{CN}$ 18-10 d'épaisseur $7 \mathrm{~mm}$. Pour une puissance de $800 \mathrm{~W}$ et une focalisation de $-0,5 \mathrm{~mm}$, de bons résultats sont obtenus à $2 \mathrm{~m} / \mathrm{mn}$ pour la forme du cordon. En revanche, des différences nettes d'attaque macrographique révèlent 1 'existence d'un mélange incomplet de deux matériaux dans la zone fondue (figuxe 2). Une analyse qualitative des espèces dans la zone à l'aide d'une.microsonde ionique (SIMS) montre que les contrastes macrographiques corxespondent bien à une hétérogénêlté de la composition chimique (figure 3), sans effet, dans le type d'application visée, sur 1a qualîtê de 1 'assemblage (étanchêỉté). Cette hẻtérogénẻỉté, due aux mouvements de convection du bain, peut cependant avoir des conséquences néfastes sur la tenue mécanique et la résistance à la corrosion des assemblages pour des couples de matériaux et/ou des critères de qualité différents:

\section{CONCLUSION}

Lès essäis đ'assemblage industriels donnent des rêsultats très encourageants, notamment pour des configurations par transparence difficiles, voir impossibles à rêaliser par d'autres procédés.

\section{REMERCIEMENTS}

Ce travail a été réalisé avec l'assistance technique de C. VIALLE (CALFETMAT) et en collaboration avec le Centre de Soudage de Framatome (CATS).

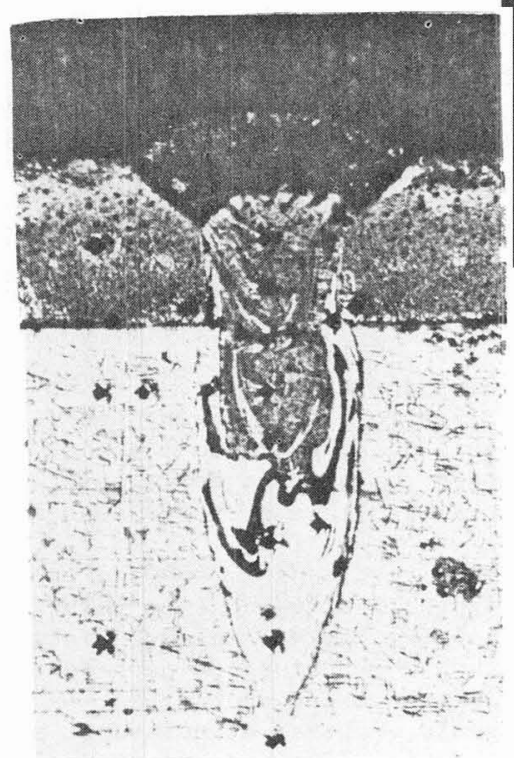

FIGURE 2
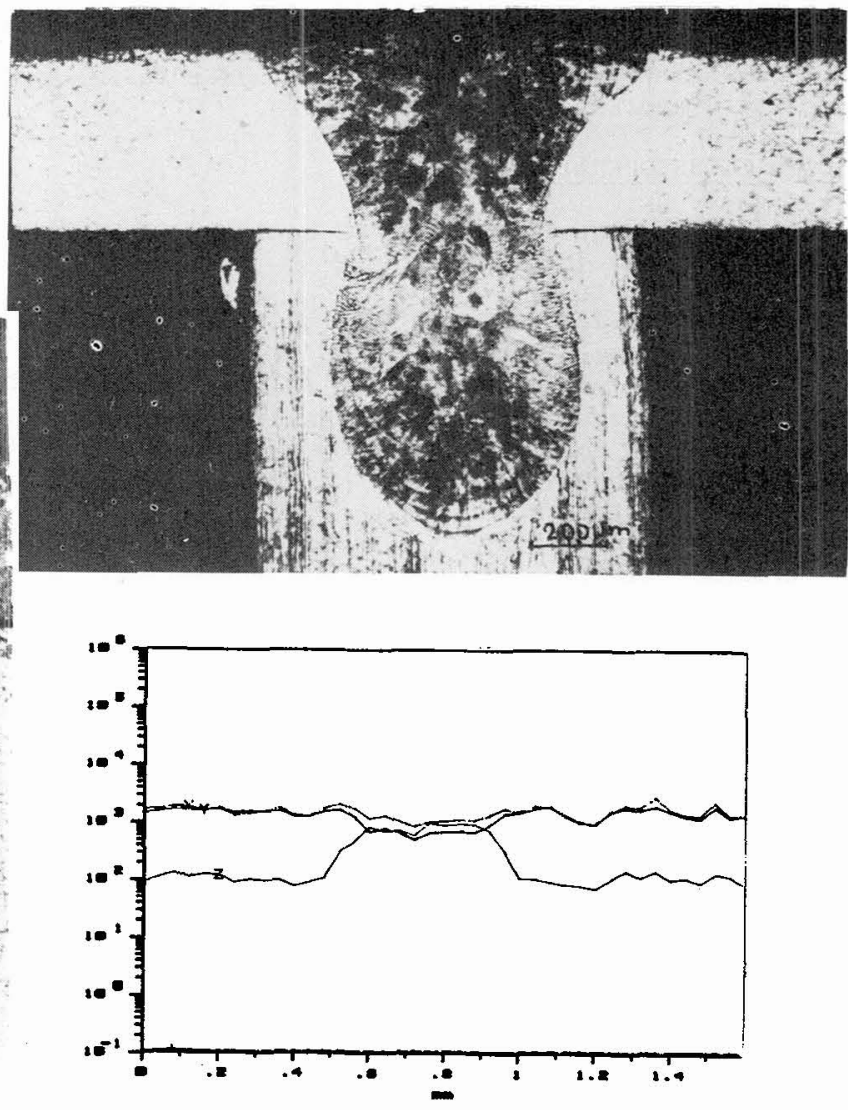

FIGURE 3 